\title{
FACTORES QUE EXPLICAN LOS BAJOS DESEMPEÑOS EN COMPETENCIAS COGNITIVAS DISCIPLINARES DE ESTUDIANTES EN EL PROGRAMA ADMINISTRACIÓN FINANCIERA*
}

\author{
FACTORS THAT EXPLAIN THE LOW PERFORMANCE IN COGNITIVE \\ DISCIPLINARY SKILLS OF STUDENTS IN THE FINANCIAL \\ ADMINISTRATION PROGRAM
}

Eduardo Amaya-Alvear ${ }^{1}$

\section{RESUMEN}

El presente trabajo es el resultado de un diagnóstico realizado en torno a las competencias cognitivas disciplinares, identificando los factores que obstaculizan el proceso de enseñanza aprendizaje de los estudiantes del programa de administración financiera (ADFU) de una universidad colombiana. El estudio asumió un enfoque metodológico de diseño cualitativo, con el cual, se pueden descubrir y definir preguntas de investigación que conducen a observar la realidad que ocurre en el entorno del programa ADFU. Se realizaron entrevistas semiestructurada y estructurada para estudiantes, una semi estructurada para profesores, y dos revisiones documentales. Se identificaron fallas de contexto educativo, patrones de comportamiento, factores afectivos y motivacionales, y deficiencias cognitivas como factores que explican los bajos desempeños de los estudiantes. Ante los anteriores resultados, se propuso un mecanismo de articulación, gestión y evaluación de las prácticas educativas frente al Proyecto Educativo Institucional, con miras a mejorar los bajos desempeños de los estudiantes en el proceso de aprendizaje del programa administración financiera (ADFU).

PAlABRAS ClaVE: Competencias financieras, Factores de desempeño, formación disciplinar, Proyecto Educativo Institucional.

\footnotetext{
* $\quad$ Proyecto auspiciado por la Corporación Universitaria Minuto de Dios UVD (Colombia), en el programa Maestría en Educación, 2018. www.uniminuto.edu

1 Corporación Universitaria Minuto de Dios - UNIMINUTO. Maestría en Educación.
} 


\begin{abstract}
The present work is the result of a diagnosis made around the disciplinary cognitive competences, identifying the factors that impede the teaching - learning process of the students of the financial administration program (ADFU) of a Colombian university. The study assumed a methodological approach to qualitative design, with which one can discover and define research questions that lead to observe the reality that occurs in the ADFU program environment. Semi-structured and structured interviews were conducted for students, a semi-structured for teachers, and two documentary reviews. Failures of educational context, behavior patterns, affective and motivational factors, and cognitive deficiencies were identified as factors that explain the low performance of students. In view of the above results, a mechanism of articulation, management and evaluation of the educational practices was proposed in front of the Institutional Educational Project, with a view to improving the low performances of the students in the learning process of the financial administration program.
\end{abstract}

KEY WORDS: Financial competences, Performance factors, disciplinary training, Institutional Educational Project.

\title{
INTRODUCCIÓN
}

El tema de la presente investigación aborda los factores que obstaculizan tener buenos desempeños en competencias financieras de estudiantes de una universidad colombiana. En educación la palabra competencia ha sido definida etimológicamente como una derivación del latín, competeré, que se identifica como la responsabilidad que tiene el individuo de utilizar sus saberes y potencialidades para cambiar realidades (Zambrano, 2011). Las competencias, representan una combinación dinámica de las capacidades cognitivas y meta cognitivas, de conocimiento y entendimiento, interpersonales, intelectuales y prácticas, así como de los valores éticos (Tuning, 2003). Desde esta perspectiva, cada curso del componente profesional del programa administración financiera de la universidad (Uniminuto, 2010), describe la competencia cognitiva de la disciplina (CCD) como la capacidad de comprender las decisiones financieras en las organizaciones y gestionar las funciones financieras propias de las mismas. Por ello, el Proyecto Educativo Institucional (PEI, 2014) de la universidad en estudio, ha condicionado su modelo educativo para que sus estudiantes en general, y los de último semestre de administración financiera en particular, se les facilite la excelencia académica y el desarrollo de habilidades y actitudes de aprendizaje autónomo en un ambiente de aprendizaje presencial y virtual. 
Sin embargo, y teniendo en cuenta lo anterior, la presente investigación se realiza porque se hacen evidentes las dificultades y problemas en los discentes para comprender los temas financieros (Corbi, 2003) relacionados con matemática financiera, análisis financiero y evaluación de proyectos. Por tanto, la formulación del problema se basó en preguntar: icuáles son los componentes de las competencias cognitivas responsables de los bajos desempeños en las competencias cognitivas disciplinares de los estudiantes de último semestre en el programa administración financiera? Al respecto Pérez (2006), expone que la comprensión de temas tratados en textos va muy relacionado con los procesos cognitivos involucrados en el pensamiento (resolución de problemas, el lenguaje, la formación de conceptos, el procesamiento de información, entre otros). Por su parte, Carretero \& Limón (1997) plantean que el razonamiento humano debe tener una lógica formal y argumentativa que facilite la comprensión de los problemas en la educación.

La forma de entender más de cerca la problemática planteada es, esperar siempre que los resultados del aprendizaje sean manifestaciones de lo que se espera que un estudiante sepa, entienda y sea capaz de demostrar una vez concluido el aprendizaje (Tuning, 2003). Por ello, este estudio de investigación trabajó con cuatro enfoques que facilitaron la identificación de factores responsables de los bajos desempeños en CCD financieras. El primero es, el enfoque institucional con el cual se abordan las fallas de contexto educativo; el segundo es, el enfoque socio-cultural con el cual se identifican patrones de comportamiento de alumnos y profesores frente a los procesos de enseñar-aprender-evaluar; el tercero es, el enfoque disciplinar que permite resaltar las deficiencias cognitivas en estudiantes, y cuarto, se halla el enfoque psicológico que permite señalar los factores afectivos y motivacionales de los estudiantes para hacer frente a la apropiación de las competencias respectivas fijadas en los cursos del programa.

Una falla de contexto educativo preponderante en la universidad, se debe a que en el desarrollo del proceso enseñanza-aprendizaje-evaluación, el estudiante se enfrenta a mucho contenido curricular y poco tiempo para desarrollar ese contenido (Ríos, 2001), produciendo una divergencia que se traduce en presentación de tareas sin la profundización de los temas y generación de campos de reflexión que permitan una práctica reflexiva. La investigación halló que, en el curso de matemática financiera un estudiante debe evacuar, en el transcurso de dos semanas, los temas y talleres de seis capítulos del libro guía (Ramírez, J., y Martínez, E., 2016) del curso, produciendo una carrera contra el tiempo, y sobre la cual, Cornella (2000) pone una voz de alerta catalogando la cantidad abruma- 
dora de información como "infoxicadora", porque se tiene más información de la que se puede manejar. Igualmente, las fallas de contexto educativo se presentan cuando no hay similitudes coherentes entre los resultados de las evaluaciones de Estado (ICFES, 2016) obtenidas por los estudiantes, y los resultados obtenidos de las evaluaciones en los cursos de componente profesional del programa ADFU de la universidad. Estas fallas de contexto educativo las identifica Díaz B. (1993), cuando plantea que la asignación de una calificación es un problema de la institución educativa y de la sociedad, no es una cuestión básicamente de la pedagogía.

De la misma forma, en el enfoque sociocultural, la investigación encontró factores actitudinales tales como la falta de espíritu investigativo en los estudiantes, los bajos niveles de disposición para construir conocimiento en forma autónoma, la falta de hábito de lectura y la poca o nula actualización de conocimientos previos (Pérez, 2006), entre otros . En este sentido, Tesouro (2015) destaca un patrón de comportamiento muy común en los estudiantes que es su falta de planificar, gestionar y evaluar sus propios procesos de aprendizaje, lo cual, coincide en su totalidad con lo hallado en la investigación, a pesar de que la misma universidad promueve autodisciplina, la autonomía y el acompañamiento tutorial presencial y virtual.

Con respecto al enfoque disciplinar, es importante la apropiación de la ciencia formal (conceptos financieros, teorías financieras, leyes y principios, fórmulas financieras, entre otros) y el lenguaje técnico basados en una lógica matemática, que debe ser una habilidad en el estudiante del programa, lo cual se manifestó en la investigación con bajos desempeños de los estudiantes en habilidades técnicas, y desempeños regulares en habilidades conceptuales. Al respecto Carretero, et al. (1997) refieren que, tradicionalmente los estudios sobre razonamiento han utilizado principalmente tareas y situaciones con contenido abstracto que han versado sobre tareas lógico-matemáticas.

Por último, el enfoque psicológico (Jover, 2011) plantea que las CCD se adquirirán siempre y cuando el estudiante abandone su zona de confort y se enfrente a la nueva información y conocimiento, buscando sus propias motivaciones que condicionan sus esfuerzos. En este aparte, la investigación halló un alto nivel de dependencia de los alumnos hacia la cátedra del profesor, para resolver los problemas de tipo financiero,

Desde la perspectiva de estos cuatro enfoques, la presente investigación analizó por qué los bajos desempeños en CCD financieras de estudiantes de último semestre, en el programa ADFU, con el fin de contemplar nuevas investigaciones y nuevos planes de mejoramiento. 


\section{MÉTODOS}

Se estableció un plan de trabajo en cinco fases. En la primera se formuló el problema conectándolo con la perspectiva teórica (Hernández S, et al., 2014) sobre los componentes de competencias cognitivas que producen bajos desempeños en CCD financieras. En una segunda fase, teniendo en cuenta cinco objetivos fijados, se determina que el tipo de estudio es de diseño cualitativo, utilizado para descubrir y definir preguntas de investigación, conducentes a observar la realidad de los bajos desempeños estudiantiles. El universo promedio poblacional es de 30 estudiantes de último semestre de ADFU y 10 profesores. La muestra (12 estudiantes y 4 profesores) es por conveniencia, focal y de tipo no probabilístico.

Para la fase 3, se implementaron categorías y subcategorías de análisis que permiten la instrumentalización para recoger información. En la Tabla 1 se refleja el diseño del trabajo instrumental.

Tabla 1

CATEGORIZACIÓN E INSTRUMENTOS

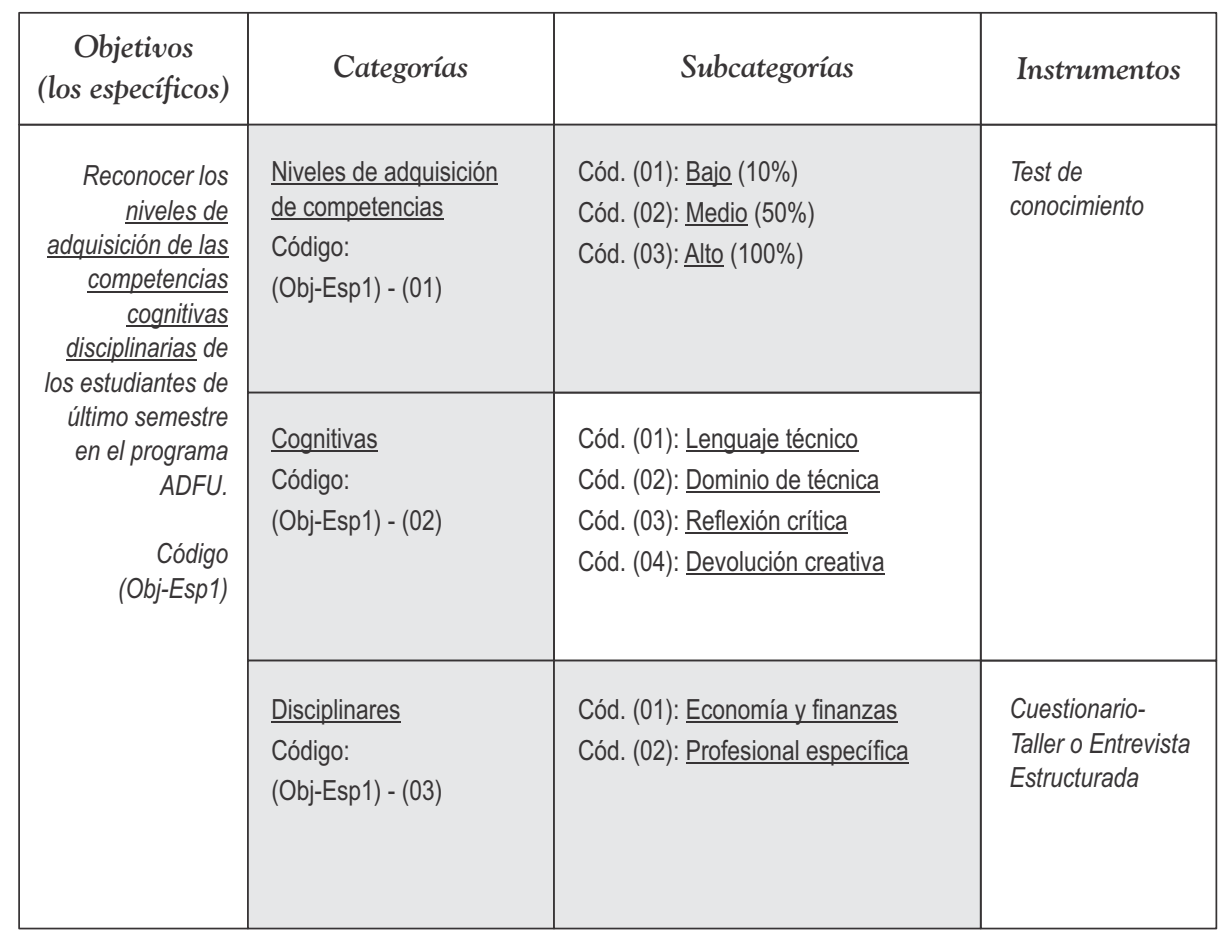


Tabla 1 (Cont.)

\begin{tabular}{|c|c|c|c|}
\hline $\begin{array}{c}\text { Objetivos } \\
\text { (los específicos) }\end{array}$ & Categorías & Subcategorías & Instrumentos \\
\hline $\begin{array}{r}\text { Comparary } \\
\text { analizar los } \\
\text { resultados de } \\
\text { pruebas } \\
\text { evaluatorias } \\
\text { estatales }\end{array}$ & $\begin{array}{l}\text { Estudiantes último } \\
\text { semestre } \\
\text { Código: } \\
\text { (Obj-Esp2) - (01) }\end{array}$ & $\begin{array}{l}\text { Cód. (01): Actitud frente a la } \\
\text { evaluación } \\
\text { Cód. (02): Comprensión de pruebas } \\
\text { evaluatorias } \\
\text { Cód. (03): Estrategias para toma de } \\
\text { decisiones en evaluación }\end{array}$ & $\begin{array}{l}\text { Entrevista abierta } \\
\text { para estudiantes }\end{array}$ \\
\hline $\begin{array}{r}\text { institucionales } \\
\text { Uniminuto, para } \\
\text { identificar } \\
\text { componentes } \\
\text { de bajos } \\
\text { desempeños en } \\
C C D \text { financieras }\end{array}$ & $\begin{array}{l}\text { Resultados Saber Pro } \\
\text { Código: } \\
\text { (Obj-Esp2) - (02) }\end{array}$ & $\begin{array}{l}\text { Cód. (01): Análisis financiero } \\
\text { Cód. (02): Análisis de inversiones y } \\
\text { financiaciones } \\
\text { Cód. (03): Proyección de estados } \\
\text { financieros } \\
\text { Cód. (04): Valoración de proyectos }\end{array}$ & \multirow[t]{2}{*}{$\begin{array}{l}\frac{\text { Revisión }}{\text { Documental: }} \\
\text { Saber Pro; } \\
\text { Plataforma } \\
\text { Banner de la } \\
\text { universidad }\end{array}$} \\
\hline $\begin{array}{r}\text { último semestre } \\
\text { ADFU. } \\
\text { Código } \\
\text { (Obj-Esp2) }\end{array}$ & $\begin{array}{l}\text { Resultados Cursos del } \\
\text { Componente Profesional } \\
\text { Código: } \\
\text { (Obj-Esp2) - (03) }\end{array}$ & $\begin{array}{l}\text { Cód. (01): Análisis financiero } \\
\text { Cód. (02): Análisis de inversiones y } \\
\text { financiaciones } \\
\text { Cód. (03): Proyección de estados } \\
\text { financieros } \\
\text { Cód. (04): Valoración de proyectos }\end{array}$ & \\
\hline $\begin{array}{r}\text { Identificar las } \\
\text { metodologias } \\
\text { implementadas por } \\
\text { los profesores para } \\
\text { que los estudiantes } \\
\text { apropien CCD } \\
\text { financieras. } \\
\text { Código (Obj-Esp3) }\end{array}$ & $\begin{array}{l}\frac{\text { Metodologías de }}{\text { enseñanza y evaluación }} \\
\text { implementadas } \\
\text { Código } \\
\text { (Obj-Esp3) - (01) }\end{array}$ & $\begin{array}{l}\text { Cód. (01): Grupales: } \\
\text { Cód. (02): Magistrales: } \\
\text { Cód. (03): Individuales o autónomas. }\end{array}$ & $\begin{array}{l}\text { Entrevistas } \\
\text { semiestructurada } \\
\text { para profesores. }\end{array}$ \\
\hline \multirow{2}{*}{$\begin{array}{r}\text { Identificar los } \\
\text { métodos de } \\
\text { aprendizaje y las } \\
\text { actitudes de los } \\
\text { estudiantes frente al } \\
\text { proceso enseñanza } \\
\text { aprendizaje que } \\
\text { validan sus } \\
\text { desempeños en } \\
\text { CCD financieras. }\end{array}$} & $\begin{array}{l}\text { Métodos de aprendizaje } \\
\text { Código } \\
\text { (Obj-Esp4) - (01) }\end{array}$ & $\begin{array}{l}\text { Cód. (01): Aprendizaje por inducción. } \\
\text { Cód. (02): Aprendizaje colaborativo. } \\
\text { Cód. (03): Aprendizaje por discusión. }\end{array}$ & \multirow[t]{2}{*}{$\frac{\frac{\text { Entrevista }}{\text { semiestructurada }}}{\text { para estudiantes. }}$} \\
\hline & $\begin{array}{l}\frac{\text { Actitud frente al }}{\text { conocimiento }} \\
\text { Código } \\
\text { (Obj-Esp4) - (02) }\end{array}$ & $\begin{array}{l}\text { Cód. (01): Activa, propositiva y } \\
\text { recursiva. } \\
\text { Cód. (02): Receptiva, pasiva y } \\
\text { cumplidora de tareas. } \\
\text { Cód. (03): Investigativa }\end{array}$ & \\
\hline
\end{tabular}


Tabla 1 (Cont.)

\begin{tabular}{|c|c|c|c|}
\hline $\begin{array}{c}\text { Objetivos } \\
\text { (los específicos) }\end{array}$ & Categorías & Subcategorías & Instrumentos \\
\hline \multirow[t]{2}{*}{$\begin{array}{r}\text { Establecer un } \\
\text { diagnóstico de } \\
\text { componentes de } \\
\text { desempeño en CCD } \\
\text { financieras en } \\
\text { estudiantes último } \\
\text { semestre de ADFU }\end{array}$} & $\begin{array}{l}\text { Factores de influencia en } \\
\text { la apropiación de CCD. } \\
\text { Código (Obj-Esp5) - (01) }\end{array}$ & $\begin{array}{l}\text { Cód. (01): Administración de la } \\
\text { información y organización del } \\
\text { conocimiento. } \\
\text { Cód. (02): } \text { Prerrequisitos de } \\
\text { conocimientos. } \\
\text { Cód. (03): Divergencia entre la } \\
\text { cantidad de contenidos y el tiempo } \\
\text { disponible para asimilarlos. } \\
\text { Cód. (04): Actitud pasiva o activa del } \\
\text { estudiante frente a la información y el } \\
\text { conocimiento. } \\
\text { Cód. (05): La práctica docente como } \\
\text { facilitadora de CCD. } \\
\text { Cód. (06): Pensamiento eficaz como } \\
\text { práctica académica facilitadora de } \\
\text { CCD. } \\
\text { Cód. (07): Pensamiento crítico como } \\
\text { práctica académica facilitadora de } \\
\text { CCD }\end{array}$ & $\begin{array}{l}\text { Entrevista } \\
\text { semiestructurada } \\
\text { para estudiantes. }\end{array}$ \\
\hline & $\begin{array}{l}\text { Manejo y aplicabilidad de } \\
\text { temas y conceptos } \\
\text { Código (Obj-Esp5) - (02) }\end{array}$ & $\begin{array}{l}\text { Cód. (01): Economía y finanzas } \\
\text { (matemática financiera y análisis } \\
\text { financiero) } \\
\text { Cód. (02): Profesional específica } \\
\text { (formulación y evaluación de } \\
\text { proyectos) }\end{array}$ & $\begin{array}{l}\text { Test de } \\
\text { conocimiento para } \\
\text { estudiantes }\end{array}$ \\
\hline
\end{tabular}

Fuente: elaboración propia.

Con fines de alcanzar los cinco objetivos de la investigación, se determinó trabajar con tres cursos (matemática financiera, análisis financiero, formulación y evaluación de proyectos) del componente profesional del programa ADFU. El primer instrumento de recolección de información es el test de conocimiento como lo muestra la tabla I, que funciona como una entrevista semiestructurada (Ryen, 2013; y King y Horrocks, 2010, citados por Hernández, 2014, p. 403) basada en una guía de asuntos o preguntas en donde el entrevistador tiene la libertad de introducir preguntas adicionales, para precisar conceptos u obtener mayor información. En este test, se hacen preguntas que buscan que el estudiante 
autoevalúe su desempeño cognitivo disciplinar como bajo (0\% - 10\%), medio $(11 \%-50 \%)$ o alto $(51 \%-100 \%)$ sobre núcleos temáticos propuestos para cada curso escogido por la investigación, y explique las causas de sus desempeños en los tres cursos escogidos por la investigación.

El segundo instrumento de recolección de información es un cuestionario para estudiantes, que tiene como objetivo identificar si el estudiante tiene claridad en aplicar los conocimientos adquiridos (conceptos financieros, teorías, leyes, principios, fórmulas financieras, técnicas, etc.) en la solución de cuestionarios y ejercicios de talleres referidos a los cursos de matemática financiera, análisis financiero y, formulación y evaluación de proyectos. Este es un instrumento guía de preguntas específicas y se sujeta exclusivamente a ella (Hernández, 2104, p 403), es una entrevista estructurada. Por cada curso, y por escrito, se realizan 5 preguntas tipo cuestionario y 5 preguntas tipo ejercicio. Con las preguntas tipo cuestionario, se exploran los conceptos financieros, teorías, leyes y principios que son manejados por los estudiantes; y con las cinco preguntas tipo ejercicio, se recogen los criterios con los cuales los estudiantes utilizan las fórmulas y técnicas para encontrar la solución a los problemas propuestos.

Como tercer instrumento de recolección de información, se ha hecho uso de la entrevista abierta para estudiantes, que se fundamenta en una guía general de contenido y el entrevistador posee toda la flexibilidad para manejarla (Hernández, 2014). A diferencia de los cuestionarios, este instrumento se basa en preguntas abiertas que brindan la flexibilidad para que el estudiante pueda explicar el porqué de los resultados que obtuvo en el proceso de enseñanzaaprendizaje-evaluación de los tres cursos escogidos en esta investigación. Adicionalmente, se pretende que el instrumento pueda identificar las particulares motivaciones o desmotivaciones del estudiante, cuando se le pone a disposición la administración autónoma de sus tiempos de estudio, la comunicación sincrónica y asincrónica con su profesor, el uso de la plataforma virtual y los elementos necesarios de ayuda didáctica. De esta forma, se pueden describir los esfuerzos que hace un estudiante particular, guiado por sus propias motivaciones, para alcanzar las metas propuestas de asimilar CCD (motivaciónesfuerzo-habilidad). Navarro (2003) (cita sacada de Covington, 1984) describe que hay tres tipos de estudiantes: los que se consideran capaces presentan alta motivación de logro y muestran confianza en sí mismos; los que aceptan el fracaso y renuncian al esfuerzo; los que evitan el fracaso, recurren a estrategias como trampas en los exámenes. 
Otros instrumentos utilizados en la investigación para recolectar información, corresponden a las revisiones documentales hechas a las pruebas de Estado Saber Pro para estudiantes de educación superior, y las calificaciones que obtuvieron los estudiantes en los tres cursos escogidos por el presente estudio. Se estableció un comparativo entre los resultados que facilitaran la identificación de similitudes y diferencias que se presentan entre ambos espacios de evaluación. Fundamentalmente, este instrumento le sirve al investigador para conocer los antecedentes de un ambiente, así como las vivencias o situaciones que se producen en él y su funcionamiento cotidiano y anormal (LeCompte y Schensul, 2013; Rafaeli y Pratt, 2012; Van Maanen, 2011; y Zemliansky, 2008, citados por Hernández, 2014, p. 415, párr. 4). Con este comparativo se revisaron las metodologías de evaluación (López, 2013) implementadas por los profesores que lideran los tres cursos de la presente investigación, encontrándose que la principal evaluación que se realiza es la heterónoma (Uniminuto, s.f., pág.14), es decir, el profesor evalúa al estudiante utilizando ciertos criterios o rúbricas como parámetros graduales de desempeño, resultando también que esta evaluación es influenciada por otros factores que son exógenos a las rúbricas fijadas.

Se hizo uso de la entrevista guía o semiestructurada para profesores, que tiene como propósito fundamental describir las prácticas usadas por ellos para facilitar la apropiación de CCD en los estudiantes, y poder cruzar esta información con las respuestas que dan los estudiantes acerca de esas metodologías. De hecho, esto permite explicar los niveles de coincidencia en la efectividad de las metodologías de enseñanza compartida, y en algunos casos, se encuentran respuestas inesperadas que entran a incorporar el abanico de posibles causas en los bajos desempeños de CCD financieras. También, el instrumento procura identificar los métodos de evaluación que son utilizados por el profesor para validar las CCD de los estudiantes. Como lo plantea el Proyecto Tuning (2003), los resultados del aprendizaje se expresan en niveles de competencia que debe conseguir el estudiante.

Finalmente, en la fase 4, luego de aplicados los instrumentos, se sistematiza la información con fines de análisis de resultados, conclusiones y recomendaciones.

\section{RESULTADOS}

A continuación se presentan los resultados de la investigación. 


\section{RESULTADOS DEL TEST DE CONOCIMIENTO PARA ESTUDIANTES}

Según la autoevaluación que hacen los estudiantes de último semestre con respecto a las CCD propuestas en el curso de matemática financiera, se observa (Tabla 2) que el grupo tiende a autoevaluar su nivel de asimilación entre bajo y medio, pudiéndose interpretar como que los estudiantes no están apropiándose plenamente de los conocimientos propuestos en este curso. Solo en el núcleo temático de interés simple e interés compuesto, más del 50\% de estudiantes (entre 7 y 10) se autoevalúan con un nivel medio de asimilación, y en el resto de núcleos temáticos se autoevalúan con un nivel bajo (más del 50\% de estudiantes), porque argumentan el poco tiempo disponible para desarrollar los temas como una práctica reflexiva. Poggioli (2005) plantea al respecto que una información o conocimiento es adquirido cuando se practica la estrategia de memoria, la cual, requiere el uso de más tiempo para estudiar y almacenar información.

Pero en el curso de análisis financiero, más del 50\% de 12 estudiantes (entre 7 y 12) autovaloran su asimilación de CCD con un nivel medio, especialmente en generalidades del análisis financiero, valor económico agregado y la determinación de las fuentes de recursos de la empresa y sus principales aplicaciones (Tabla 3). Principalmente, estas afirmaciones obedecen a que los estudiantes encuestados manifiestan que tienen la oportunidad de interpretar la información suministrada en el curso, relacionándola con sus actividades laborales diarias, y constatar los conocimientos previos laborales con los adquiridos en la academia. Sin embargo, y contrario a lo expresado por los discentes, Díaz B. (1983) plantea que no es claro en el alumno (y supuestamente en el docente), que la actividad en el aprendizaje, en un primer momento, está conformada por "acciones internas" del sujeto que se coloca a sí mismo ante situaciones de trabajar con un objeto de estudio determinado.

Y, en cuanto al curso de formulación y evaluación de proyectos, la tendencia de autovaloración de los estudiantes (Tabla 4) es hacia una asimilación de CCD entre baja y media (como sucede en el curso de matemática financiera). Lo relevante en este curso es que ninguno de los estudiantes apunta a autoevaluar su nivel de asimilación como alto, y esto tiene que ver, como lo afirman ellos, con las dificultades para definir la problemática de la evaluación de proyectos (10 de los 12 estudiantes lo exponen), porque no están enseñados a realizar ese tipo de ejercicios; además, porque tienen dificultades conceptuales y procedimentales (la totalidad de los estudiantes encuestados lo manifiestan) para identificar variables financieras que les permitan construir modelos matemáticos con qué evaluar financieramente y realizar análisis de riesgo y sensibilidad financiera. 
Tabla 2

\section{AUTOEVALUACIÓN DE DESEMPEÑO EN COMPETENCIAS COGNITIVAS DISCIPLINARES POR PARTE DE ESTUDIANTES DE ÚLTIMO SEMESTRE}

\section{CURSO MATEMÁTICA FINANCIERA}

\begin{tabular}{|c|c|c|c|c|}
\hline \multirow[t]{2}{*}{ Núcleo temático } & \multirow[t]{2}{*}{ Competencia Cognitiva Disciplinar } & \multicolumn{3}{|c|}{$\begin{array}{l}\text { Valoración del grado } \\
\text { de asimilación }\end{array}$} \\
\hline & & Baja & Media & Alta \\
\hline \multirow{3}{*}{$\begin{array}{l}\text { Interés Simple e } \\
\text { Interés Compuesto }\end{array}$} & $\begin{array}{l}\text { Identificar las diferentes clases de interés que existen dentro } \\
\text { de la matemática financiera con el propósito de utilizarlos } \\
\text { para encontrar el valor del dinero a través del tiempo. }\end{array}$ & 2 & 7 & 3 \\
\hline & $\begin{array}{l}\text { Construir diagramas económicos donde se puedan graficar } \\
\text { los diferentes elementos que integran un ejercicio } \\
\text { matemático. }\end{array}$ & 2 & 7 & 3 \\
\hline & $\begin{array}{l}\text { Encontrar valores presentes y valores futuros a partir de un } \\
\text { monto de dinero determinado. }\end{array}$ & 0 & 10 & 2 \\
\hline \multirow{2}{*}{$\begin{array}{l}\text { Tasas de interés } \\
\text { nominal y efectiva; } \\
\text { rentabilidad }\end{array}$} & $\begin{array}{l}\text { Diferenciar entre tasas nominales y efectivas; entre vencidas } \\
\text { y anticipadas. Medir rentabilidad. }\end{array}$ & 5 & 5 & 2 \\
\hline & $\begin{array}{l}\text { Conocer las conversiones de las tasas nominales y efectivas } \\
\text { a diferentes periodos. }\end{array}$ & 6 & 4 & 2 \\
\hline \multirow{2}{*}{ Anualidades } & $\begin{array}{l}\text { Reconocer la utilidad que tienen las anualidades en el } \\
\text { desarrollo de las distintas técnicas utilizadas en la } \\
\text { matemática financiera para la toma de decisiones. }\end{array}$ & 7 & 3 & 2 \\
\hline & $\begin{array}{l}\text { Conocer correctamente sus fórmulas y aplicabilidad en la } \\
\text { matemática financiera para la toma de decisiones. }\end{array}$ & 7 & 4 & 1 \\
\hline \multirow{2}{*}{$\begin{array}{l}\text { Financiación y } \\
\text { amortización }\end{array}$} & $\begin{array}{l}\text { Identificar los diferentes sistemas de financiación y } \\
\text { amortización. }\end{array}$ & 11 & 1 & 0 \\
\hline & $\begin{array}{l}\text { Calcular correctamente los problemas planteados de } \\
\text { financiación y amortización, a través de fórmulas, calculadora } \\
\text { y hoja electrónica de cálculo. }\end{array}$ & 11 & 1 & 0 \\
\hline $\begin{array}{l}\text { Inversión e } \\
\text { instrumentos de } \\
\text { evaluación } \\
\text { financiera }\end{array}$ & $\begin{array}{l}\text { Saber identificar y aplicar los instrumentos de evaluación } \\
\text { financiera VAN, TIR, RBC, CAUE, EVA. }\end{array}$ & 11 & 1 & 0 \\
\hline
\end{tabular}

Fuente: Elaboración propia con base en las guías curriculares de cada curso. 2017 y respuestas de estudiantes. 
Tabla 3

\section{AUTOEVALUACIÓN DE DESEMPEÑO EN COMPETENCIAS COGNITIVAS DISCIPLINARES POR PARTE DE ESTUDIANTES DE ÚLTIMO SEMESTRE}

\section{CURSO ANÁlisis FINANCIERO}

\begin{tabular}{|c|c|c|c|c|}
\hline \multirow[t]{2}{*}{ Núcleo temático } & \multirow[t]{2}{*}{ Competencia Cognitiva Disciplinar } & \multicolumn{3}{|c|}{$\begin{array}{l}\text { Valoración del grado } \\
\text { de asimilación }\end{array}$} \\
\hline & & Baja & Media & Alta \\
\hline \multirow{2}{*}{$\begin{array}{l}\text { Generalidades del } \\
\text { análisis financiero }\end{array}$} & Identificar y asimilar el concepto de la función financiera. & 0 & 9 & 3 \\
\hline & $\begin{array}{l}\text { Reconocer los beneficios y oportunidades que ofrece la } \\
\text { función financiera. }\end{array}$ & 0 & 12 & 0 \\
\hline \multirow{2}{*}{$\begin{array}{l}\text { Estructura y } \\
\text { evolución financiera } \\
\text { de la empresa }\end{array}$} & $\begin{array}{l}\text { Asimilar los tipos de análisis a los estados financieros más } \\
\text { utilizados. }\end{array}$ & 2 & 7 & 3 \\
\hline & $\begin{array}{l}\text { Reconocer la importancia de un correcto análisis a los } \\
\text { estados financieros para la toma de decisiones en las } \\
\text { empresas. }\end{array}$ & 0 & 12 & 0 \\
\hline \multirow{2}{*}{$\begin{array}{l}\text { Indicadores } \\
\text { financieros }\end{array}$} & $\begin{array}{l}\text { Asimilar los diferentes indicadores financieros y su aplicación } \\
\text { para un adecuado análisis de los estados financieros. }\end{array}$ & 2 & 10 & 0 \\
\hline & $\begin{array}{l}\text { Reconocer la clasificación de los indicadores financieros y la } \\
\text { importancia de la misma. }\end{array}$ & 0 & 12 & 0 \\
\hline \multirow{2}{*}{$\begin{array}{l}\text { Valor Económico } \\
\text { Agregado - EVA }\end{array}$} & $\begin{array}{l}\text { Identificar técnicas para la evaluación del desempeño de una } \\
\text { empresa. }\end{array}$ & 0 & 12 & 0 \\
\hline & $\begin{array}{l}\text { Asimilar cómo se puede determinar el valor agregado de las } \\
\text { empresas. }\end{array}$ & 0 & 12 & 0 \\
\hline \multirow{2}{*}{$\begin{array}{l}\text { Determinación de } \\
\text { las fuentes de } \\
\text { recursos de la } \\
\text { empresa y sus } \\
\text { principales } \\
\text { aplicaciones }\end{array}$} & $\begin{array}{l}\text { Identificar técnicas para determinar las fuentes de recursos } \\
\text { en una empresa. }\end{array}$ & 0 & 12 & 0 \\
\hline & $\begin{array}{l}\text { Implementar técnicas para determinar los diferentes usos que } \\
\text { se les dan a los recursos de la empresa. }\end{array}$ & 0 & 12 & 0 \\
\hline
\end{tabular}

Fuente: Elaboración propia con base en las guías curriculares de cada curso. 2017 y respuestas de estudiantes. 
Tabla 4

AUTOEVALUACIÓN DE DESEMPEÑO EN COMPETENCIAS COGNITIVAS DISCIPLINARES POR PARTE DE ESTUDIANTES DE ÚLTIMO SEMESTRE

CURSO FORMULACIÓN Y EVALUACIÓN DE PROYECTOS

\begin{tabular}{|c|c|c|c|c|}
\hline \multirow[t]{2}{*}{ Núcleo temático } & \multirow[t]{2}{*}{ Competencia Cognitiva Disciplinar } & \multicolumn{3}{|c|}{$\begin{array}{l}\text { Valoración del grado } \\
\text { de asimilación }\end{array}$} \\
\hline & & Baja & Media & Alta \\
\hline \multirow{3}{*}{$\begin{array}{l}\text { Generalidades, } \\
\text { importancia y } \\
\text { tipología de los } \\
\text { proyectos }\end{array}$} & Definir la problemática de la evaluación de proyectos. & 10 & 2 & 0 \\
\hline & $\begin{array}{l}\text { Identificar la utilidad que revisten los proyectos de inversión } \\
\text { en la sociedad. }\end{array}$ & 0 & 12 & 0 \\
\hline & $\begin{array}{l}\text { Diferenciar los proyectos de inversión privada y los proyectos } \\
\text { de inversión pública. }\end{array}$ & 0 & 12 & 0 \\
\hline $\begin{array}{l}\text { Formulación y } \\
\text { obtención de } \\
\text { información }\end{array}$ & $\begin{array}{l}\text { Identificar las variables de los estudios de mercado, técnico, } \\
\text { organizacional y legal. }\end{array}$ & 7 & 5 & 0 \\
\hline $\begin{array}{l}\text { Sistematización } \\
\text { de variables } \\
\text { financieras }\end{array}$ & $\begin{array}{l}\text { Integrar las variables identificadas como variables financieras } \\
\text { con fines de ofrecer información de salida para la evaluación } \\
\text { de los proyectos }\end{array}$ & 9 & 3 & 0 \\
\hline \multirow{3}{*}{$\begin{array}{l}\text { Evaluación de } \\
\text { proyectos de } \\
\text { inversión privada }\end{array}$} & $\begin{array}{l}\text { Evaluar los proyectos utilizando los instrumentos de } \\
\text { evaluación financiera VAN, TIR, RBC, CAUE, EVA. }\end{array}$ & 6 & 6 & 0 \\
\hline & $\begin{array}{l}\text { Realizar análisis de sensibilidad financiera a través de } \\
\text { escenarios. }\end{array}$ & 12 & 0 & 0 \\
\hline & Realizar análisis de riesgos financieros. & 12 & 0 & 0 \\
\hline
\end{tabular}

Fuente: Elaboración propia con base en las guías curriculares de cada curso. 2017 y respuestas de estudiantes.

Se puede interpretar que en términos generales, la categoría de asimilación de CCD en los cursos de matemática financiera, análisis financiero y, formulación y evaluación de proyectos está entre un nivel bajo y medio, y cuyo principal componente de bajos desempeños se debe a la premura del tiempo para desarrollar los temas propuestos en los planes de curso, lo que no permite superar las deficiencias conceptuales y procedimentales en las áreas de conocimiento financiero, constituyéndose en una falla de contexto educativo. 


\section{RESULTADOS DEL CUESTIONARIO PARA ESTUDIANTES}

Los bajos desempeños en CCD financieras de los estudiantes que accedieron a la entrevista (Tablas 5 y 6 ), se han hecho visibles porque frente a las preguntas cuestionario y las preguntas ejercicio hechas en los tres cursos, que pretenden identificar si se han asimilado los conocimientos y las habilidades, los discentes muestran en la mayoría de los casos, casi nula ( 0 preguntas interpretadas de 5 realizadas) interpretación de teorías y conceptos, nulo ( 0 preguntas desarrolladas de 5 realizadas) o poco desarrollo temático y procedimental, y un nulo (0 de 5) o ínfimo número de resultados acertados tanto en cuestionarios como en ejercicios.

Tabla 5

ENTREVISTA ESTRUCTURADA A TRAVÉS DE PREGUNTAS CUESTIONARIO Y DE EJERCICIOS FINANCIEROS, PARA ESTUDIANTES DE ÚLTIMO SEMESTRE ADFU ESTUDIANTE 1

\begin{tabular}{|c|c|c|c|c|c|c|}
\hline \multirow{2}{*}{$\begin{array}{l}\text { Categoría } \\
\text { de análisis }\end{array}$} & \multicolumn{2}{|c|}{ Matemática financiera } & \multicolumn{2}{|c|}{ Análisis financiero } & \multicolumn{2}{|c|}{ Evaluación de proyectos } \\
\hline & $\begin{array}{l}\text { Preguntas } \\
\text { cuestionario }\end{array}$ & $\begin{array}{l}\text { Preguntas } \\
\text { ejercicios }\end{array}$ & $\begin{array}{l}\text { Preguntas } \\
\text { cuestionario }\end{array}$ & $\begin{array}{l}\text { Preguntas } \\
\text { ejercicios }\end{array}$ & $\begin{array}{l}\text { Preguntas } \\
\text { cuestionario }\end{array}$ & $\begin{array}{l}\text { Preguntas } \\
\text { ejercicios }\end{array}$ \\
\hline Interpretación & 0 de 5 & 0 de 5 & 5 de 5 & 0 de 5 & 5 de 5 & 0 de 5 \\
\hline Desarrollo & 0 de 5 & 0 de 5 & 1 de 5 & 0 de 5 & 0 de 5 & 0 de 5 \\
\hline Resultado & 0 de 5 & 0 de 5 & 5 de 5 & 0 de 5 & 2 de 5 & 0 de 5 \\
\hline $\begin{array}{l}\text { Estrategia } \\
\text { aprendizaje }\end{array}$ & Ninguna & Ninguna & $\begin{array}{l}\text { Comparar } \\
\text { acad. /trab. }\end{array}$ & $\begin{array}{l}\text { Comparar } \\
\text { acad. /trab. }\end{array}$ & Ninguna & Ninguna \\
\hline $\begin{array}{l}\text { Componente de } \\
\text { bajo desempeño }\end{array}$ & $\begin{array}{c}\text { No me } \\
\text { gusta leer }\end{array}$ & $\begin{array}{l}\text { Deficiencia } \\
\text { conceptual }\end{array}$ & $\begin{array}{l}\text { No me } \\
\text { gusta leer }\end{array}$ & $\begin{array}{c}\text { Temas } \\
\text { superficiales }\end{array}$ & $\begin{array}{c}\text { No me } \\
\text { gusta leer }\end{array}$ & $\begin{array}{l}\text { Deficiencia } \\
\text { conceptual }\end{array}$ \\
\hline Lenguaje técnico & Muy poco & Regular & Regular & Regular & Regular & Muy poco \\
\hline $\begin{array}{l}\text { Dominio de } \\
\text { técnica }\end{array}$ & $\begin{array}{l}\text { No recuerdo } \\
\text { la técnica }\end{array}$ & $\begin{array}{l}\text { No recuerdo } \\
\text { la técnica }\end{array}$ & Poco & Poco & Regular & Poco \\
\hline $\begin{array}{l}\text { Reflexión } \\
\text { crítica }\end{array}$ & $\begin{array}{c}\text { Falta de } \\
\text { compromiso }\end{array}$ & $\begin{array}{l}\text { Soy poco } \\
\text { investigativa }\end{array}$ & $\begin{array}{l}\text { Falta de } \\
\text { compromiso }\end{array}$ & Practico poco & $\begin{array}{c}\text { Soy poco } \\
\text { investigativa }\end{array}$ & $\begin{array}{c}\text { Falta de } \\
\text { compromiso }\end{array}$ \\
\hline
\end{tabular}

Fuente: Elaboración propia con base en la entrevista estructurada para estudiantes. 
Tabla 6

ENTREVISTA ESTRUCTURADA A TRAVÉS DE PREGUNTAS

CUESTIONARIO Y DE EJERCICIOS FINANCIEROS, PARA

ESTUDIANTES DE ÚLTIMO SEMESTRE ADFU

ESTUDIANTE 2

\begin{tabular}{|c|c|c|c|c|c|c|}
\hline \multirow{2}{*}{$\begin{array}{l}\text { Categoría } \\
\text { de análisis }\end{array}$} & \multicolumn{2}{|c|}{ Matemática financiera } & \multicolumn{2}{|c|}{ Análisis financiero } & \multicolumn{2}{|c|}{ Evaluación de proyectos } \\
\hline & $\begin{array}{l}\text { Preguntas } \\
\text { cuestionario }\end{array}$ & $\begin{array}{l}\text { Preguntas } \\
\text { ejercicios }\end{array}$ & $\begin{array}{c}\text { Preguntas } \\
\text { cuestionario }\end{array}$ & $\begin{array}{l}\text { Preguntas } \\
\text { ejercicios }\end{array}$ & $\begin{array}{l}\text { Preguntas } \\
\text { cuestionario }\end{array}$ & $\begin{array}{l}\text { Preguntas } \\
\text { ejercicios }\end{array}$ \\
\hline Interpretación & 0 de 5 & 1 de 5 & 5 de 5 & 0 de 5 & 0 de 5 & 0 de 5 \\
\hline Desarrollo & 0 de 5 & 1 de 5 & 1 de 5 & 0 de 5 & 0 de 5 & 0 de 5 \\
\hline Resultado & 3 de 5 & 0 de 5 & 5 de 5 & 0 de 5 & 0 de 5 & 0 de 5 \\
\hline $\begin{array}{l}\text { Estrategia } \\
\text { aprendizaje }\end{array}$ & Ninguna & Ninguna & Ninguna & Ninguna & Ninguna & Ninguna \\
\hline $\begin{array}{l}\text { Componente } \\
\text { de bajo } \\
\text { desempeño }\end{array}$ & $\begin{array}{c}\text { Bajo } \\
\text { conocimiento } \\
\text { técnico }\end{array}$ & $\begin{array}{c}\text { Practico } \\
\text { poco } \\
\text { resolver } \\
\text { problemas }\end{array}$ & $\begin{array}{c}\text { Poco tiempo } \\
\text { para temas }\end{array}$ & $\begin{array}{c}\text { Mucha } \\
\text { teoría, poca } \\
\text { práctica }\end{array}$ & $\begin{array}{c}\text { Poco tiempo } \\
\text { para temas }\end{array}$ & $\begin{array}{c}\text { Mucha } \\
\text { teoría, poca } \\
\text { práctica }\end{array}$ \\
\hline Lenguaje técnico & Bueno & Regular & Bueno & Regular & Bueno & Regular \\
\hline $\begin{array}{l}\text { Dominio de } \\
\text { técnica }\end{array}$ & $\begin{array}{l}\text { No recuerdo } \\
\text { la técnica }\end{array}$ & $\begin{array}{l}\text { No recuerdo } \\
\text { la técnica }\end{array}$ & Poco & Poco & Poco & Nada \\
\hline $\begin{array}{l}\text { Reflexión } \\
\text { crítica }\end{array}$ & $\begin{array}{l}\text { Falta } \\
\text { profundizar } \\
\text { temas }\end{array}$ & $\begin{array}{l}\text { No soy } \\
\text { autónomo }\end{array}$ & $\begin{array}{l}\text { Hay poca } \\
\text { práctica de } \\
\text { los temas }\end{array}$ & $\begin{array}{l}\text { No soy } \\
\text { autónomo }\end{array}$ & $\begin{array}{c}\text { No tengo } \\
\text { memoria de } \\
\text { largo plazo }\end{array}$ & $\begin{array}{c}\text { Soy un } \\
\text { cumplidor de } \\
\text { tareas }\end{array}$ \\
\hline
\end{tabular}

Fuente: Elaboración propia con base en la entrevista estructurada para estudiantes.

En este sentido Cotton (1989) plantea que las competencias son un proceso de adquirir conocimiento y habilidad, que necesitan ser retenidas y fortalecidas en el tiempo, si no, dejan de ser aprendizaje.

Los componentes (Tablas 5 y 6 ) que explican los anteriores resultados son:

- Los estudiantes no construyen, por iniciativa propia, estrategias de aprendizaje que les marquen la ruta para obtener mayor conocimiento y habilidad sobre los temas financieros. 
- El hábito de lectura es bajo, y se aduce que hay poco tiempo para profundizar en los temas financieros. En este aspecto, Bautista (2013, pág.XII) plantea que, la presentación rápida y eficiente de resultados digeridos por otros se impone sobre la curiosidad que puedan suscitar los fundamentos de lo que se aprende.

- Poco se maneja el lenguaje técnico de los temas tratados, elemento que dificulta la comprensión de los cuestionarios y ejercicios.

- No se dominan las técnicas financieras, porque el estudiante asume una actitud pasiva frente a su aprendizaje: no repite los temas y ejercicios, no subraya lo importante, no realiza esquemas y modelos, hace pocas preguntas y se participa poco en la generación de nuevo conocimiento.

- No hay un comportamiento autónomo en el proceso de aprendizaje, se depende mucho de la cátedra del profesor. Para Villavicencio (2004) logramos la autonomía intelectual cuando contrastamos nuestros puntos de vista, damos sentido a nuestras construcciones, fundamentamos nuestros razonamientos y opiniones, y negociamos soluciones a determinados problemas.

\section{RESULTADOS DE LA ENTREVISTA ABIERTA PARA ESTUDIANTES}

Según los estudiantes, las prácticas pedagógicas de los profesores en el proceso de enseñanza-aprendizaje-evaluación, influyen significativamente en sus bajos desempeños de CCD financieras, porque por ejemplo, para 5 de 12 estudiantes entrevistados, las evaluaciones que hacen los profesores son muy permisivas con los estudiantes que no se esfuerzan por aprender y adquirir habilidades en finanzas, generando zonas de confort en donde no se motiva al alumno para que realice esfuerzos en el aprendizaje. Pese a estas respuestas, el modelo educativo de la universidad objeto de estudio, le propone al estudiante cuatro principios a seguir (la guía de curso como mediador pedagógico, los materiales de apoyo, el tutor como facilitador del proceso y, el contexto como ambiente de aprendizaje), de los cuales, los estudiantes solo destacan la responsabilidad del tercer principio (el tutor como facilitador del proceso).

Lo anterior, marca una actitud en el estudiante que lleva a reflexionar sobre los niveles de autoestima y motivación, esfuerzo y compromiso, responsabilidad y liderazgo frente a su propio proceso de aprendizaje, toda vez que el modelo de 
educación de la universidad procura el trabajo autónomo del estudiante y aún más, bajo la metodología de este modelo, el estudiante debe buscar sus propias motivaciones y autoestima. La autoestima puede definirse como una fuerza interna que impulsa a la persona a desarrollarse, a poner en práctica sus capacidades de manera que se vaya orientando hacia un equilibrio personal y en ocasiones puede ser causa y consecuencia de la manera en que se comportan las personas en distintas situaciones de la vida (Ribeiro, 2006, como se citó en Cantú, 2006).

\section{RESULTADOS DE LA REVISIÓN DOCUMENTAL PRUEBAS DE ESTADO}

Para este análisis de resultados el Instituto Colombiano para Evaluación de Educación Superior ICFES (entidad del Estado que realiza las pruebas), utiliza los "niveles de desempeño" como una descripción cualitativa de las competencias de los estudiantes en cada módulo (gestión financiera; formulación, evaluación y gestión de proyectos; gestión de organizaciones), fijando 4 niveles, de los cuales, el nivel 4 indica el mayor desempeño alcanzado por un estudiante en las pruebas. Para el módulo de Gestión Financiera (Tabla 7), el 70\% de los estudiantes que presentaron las pruebas, se ubican en el nivel 1, lo que indica según Guía de Orientación del Módulo (2017), que podrían conocer los conceptos contables y financieros que le permitan comprender la situación de la organización. Sin embargo, este nivel representa el más bajo desempeño de los estudiantes en las pruebas. Por su parte, el $30 \%$ de los estudiantes alcanzaron el nivel 2 con un rango de puntos entre 136 y 155 . Se interpreta que, además de lo descrito en el nivel

Tabla 7

NIVELES DE DESEMPEÑO

MÓDULO GESTIÓN FINANCIERA

\begin{tabular}{clcc}
\hline Nivel & Rango & $\begin{array}{c}\text { Número de estudiantes } \\
\text { por nivel }\end{array}$ & Porcentaje \\
\hline Nivel 1 & De 0 a 135 & 7 & $70 \%$ \\
Nivel 2 & De 136 a 155 & 3 & $30 \%$ \\
Nivel 3 & De 156 a 195 & 0 & $0 \%$ \\
Nivel 4 & De 196 a 300 & 0 & $0 \%$ \\
Total & & 10 & 100 \\
\hline
\end{tabular}

Fuente: Guía Orientación Módulo Gestión Financiera Saber Pro 2017;

Vicerrectoría General Académica Universitaria. 
anterior, el estudiante que se ubica en este nivel analiza situaciones de operación, inversión y financiación con base en la interpretación de indicadores financieros. No obstante, ambos grupos de estudiantes se ubican por debajo de la media de resultados obtenidos en el módulo.

Con respecto al Módulo Gestión de Organizaciones, los resultados de los estudiantes en las Pruebas Saber Pro son como siguen en la Tabla 8.

Tabla 8

NIVELES DE DESEMPEÑO

MÓDULO GESTIÓN DE ORGANIZACIONES

\begin{tabular}{llcc}
\hline Nivel & Rango & $\begin{array}{c}\text { Numero de estudiantes } \\
\text { por nivel }\end{array}$ & Porcentaje \\
\hline Nivel 1 & De 0 a 125 & 5 & $50 \%$ \\
Nivel 2 & De 126 a 160 & 3 & $30 \%$ \\
Nivel 3 & De 161 a 200 & 2 & $20 \%$ \\
Nivel 4 & De 201 a 300 & 0 & $0 \%$ \\
Total & & 10 & $100 \%$ \\
\hline
\end{tabular}

Fuente: Guía Orientación Módulo Gestión de Organizaciones Saber Pro 2017; Vicerrectoría General Académica Universitaria.

El 50\% de los estudiantes podrían entender situaciones particulares al interior de las organizaciones, en las que intervienen pocas variables, reconociendo conceptos propios de la administración. Este nivel de desempeño es el más bajo obtenido por los estudiantes, según el Módulo de Orientación de Competencias Gestión de Organizaciones Saber Pro 2017. También se puede observar que ningún estudiante alcanza el nivel 4 en las pruebas del módulo, lo que se interpreta como la falta de evaluar y seleccionar alternativas de decisión considerando condiciones, funciones y procesos de la organización ante diversos escenarios organizacionales; tampoco alcanzan a integrar elementos de la gestión en el análisis de múltiples situaciones organizacionales.

Por último, para analizar los resultados del Módulo Formulación, evaluación y gestión de proyectos, no se encontraron oficialmente las guías (niveles numéricos) que permitan fijar en ellas los resultados obtenidos por los estudiantes. Sin embargo, se asume en este estudio que los rangos establecidos por el 
ICFES son cualitativos, lo cual, permite que se usen para efectos de análisis, los rangos del Módulo Gestión Financiera. Siendo así, los resultados de las pruebas en el módulo pueden presentarse en los siguientes rangos (Tabla 9).

Tabla 9

NIVELES DE DESEMPEÑO

MÓDULO FORMULACIÓN, EVALUACIÓN Y GESTIÓN DE PROYECTOS

\begin{tabular}{llcc}
\hline Nivel & $\begin{array}{c}\text { Rango de } \\
\text { calificación }\end{array}$ & $\begin{array}{c}\text { Número de estudiantes } \\
\text { por nivel }\end{array}$ & Porcentaje \\
\hline Nivel 1 & De 0 a 135 & 6 & $60 \%$ \\
Nivel 2 & De 136 a 155 & 3 & $30 \%$ \\
Nivel 3 & De 156 a 195 & 1 & $10 \%$ \\
Nivel 4 & De 196 a 300 & 0 & $0 \%$ \\
Total & & 10 & $100 \%$ \\
\hline
\end{tabular}

Fuente: Guía Orientación Módulo Gestión Financiera Saber Pro 2017; Guía

Orientación Módulo Formulación, evaluación y gestión de proyectos Saber Pro 2017;

Vicerrectoría General Académica Universitaria.

El 90\% de los estudiantes que presentaron las pruebas, se ubican por debajo de la media en el Módulo Formulación, evaluación y gestión de proyectos, siendo los niveles 1 y 2 como los más bajos. Según las guías de las Pruebas Saber Pro, en el módulo se evalúan las capacidades del estudiante para formular, evaluar, ejecutar y gestionar proyectos. Ello, puede interpretarse en el presente estudio como las dificultades que tienen los estudiantes para llevar a cabo las competencias del módulo, evidenciando problemas en el diagnóstico de los contextos de los proyectos, dificultades en la realización de los estudios y otros que giran en torno a la evaluación y gestión de los mismos.

\section{RESULTADOS DE LA REVISIÓN DOCUMENTAL CALIFICACIONES ESTUDIANTES ADFU}

La revisión documental que se ha alcanzado hasta el momento con respecto a las calificaciones que obtuvieron los estudiantes de la muestra en los cursos Matemática Financiera, Análisis Financiero y Formulación y Evaluación de Proyectos, solo ha sido posible para el primero porque el profesor del curso permitió acceder a esa base de datos siempre y cuando se conservara la confidencialidad de 
la misma. Para este análisis, se ha emulado los cuatro niveles de desempeño propuestos por las pruebas Saber Pro, adaptando los resultados de las calificaciones a los siguientes rangos (Ver Tabla 10).

Tabla 10

NNIVELES DE DESEMPEÑO DE ESTUDIANTES DE ÚLTIMO SEMESTRE DE ADFU EN EL CURSO MATEMÁTICA FINANCIERA

\begin{tabular}{llcc}
\hline Nivel & Rango & $\begin{array}{c}\text { Número de estudiantes } \\
\text { por nivel }\end{array}$ & Porcentaje \\
\hline Nivel 1 & De 0,0 a 2,0 & 0 & $0 \%$ \\
Nivel 2 & De 2,1 a 3,5 & 0 & $0 \%$ \\
Nivel 3 & De 3,6 a 4,4 & 9 & $75 \%$ \\
Nivel 4 & De 4,5 a 5,0 & 3 & $25 \%$ \\
Total & & 12 & $100 \%$ \\
\hline
\end{tabular}

Fuente: Elaboración propia con base en la Plataforma GÉNESIS y base de datos del profesor en cabeza del curso Matemática Financiera.

El 75\% de los estudiantes han obtenido un nivel 3, que significa entre aceptable y bueno, mientras que el $25 \%$ entran en la categoría 4 , o sea, muy bueno y excelente. Estos resultados pueden interpretarse como que el 100\% de estudiantes han asimilado aceptablemente los conocimientos correspondientes a los núcleos temáticos de interés simple e interés compuesto, tasas de interés nominal y efectivo, las anualidades, la financiación y amortización, y finalmente los temas de inversión e instrumentos de evaluación financiera propuestos todos en el plan de trabajo del curso.

\section{RESULTADOS DE LA ENTREVISTA SEMI ESTRUCTURADA PARA PROFESORES}

Según el instrumento aplicado, los profesores entrevistados tienen el método de casos como la principal metodología de acompañamiento en el desarrollo de las tutorías. El método de casos (Uniminuto, s.f, pág.27) es un modo de enseñanza en el que los estudiantes construyen su aprendizaje a partir del análisis y discusión de experiencias y situaciones de la vida real. Por ejemplo, una de las profesoras que respondió la entrevista trabaja los temas de finanzas como la matemática financiera, procurando que sus estudiantes puedan hacer buena lectura 
de los extractos bancarios, tarjetas de crédito, que realicen su presupuesto familiar, que aprendan a diferenciar los distintos métodos de financiación y amortización para que puedan tomar las decisiones que más les convengan. Otra profesora pone en foros virtuales y en debates presenciales casos económicos y financieros como la inflación, para que los estudiantes puedan construir reflexiones y análisis alrededor del tema. Una tercera profesora, valiéndose del enfoque praxeológico de la universidad, procura realizar foros y glosarios de casos empresariales específicos que conlleven a que los estudiantes puedan discernir los temas y apropiar el lenguaje técnico del programa administración financiera.

Por tanto, lo que se observa en este análisis metodológico seguido por los profesores es que enseñan a través de la guía de curso, acudiendo a preguntas guías y estrategias grupales como los foros virtuales y debates presenciales, y en ello se les va todo el tiempo programado como duración del curso.

Abordando la pregunta del proceso y las finalidades principales que persigue el profesor en las evaluaciones de sus alumnos, se encuentra que todos realizan una evaluación diagnóstica o de entrada, con fines de determinar los niveles de conocimientos previos para emprender nuevas temáticas o paradigmas. Ello permite a los profesores del programa ADFU sugerirle al estudiante rutas de estudio específicas y estrategias de aprendizaje para actualizar los conocimientos.

Por el lado de las evaluaciones formativas, los profesores utilizan como instrumentos de evaluación las guías de observación, los cuestionarios y talleres, el portafolio de evidencias, la evaluación por rúbricas, entre otros, retroalimentando a los estudiantes sobre los resultados alcanzados con el fin de que valoren y aprendan de los errores cometidos en el proceso de enseñanza-aprendizaje. Estas evaluaciones que permiten determinar si un estudiante debe ser promovido a un nuevo nivel (semestre posterior), están siendo influenciadas por aspectos exógenos a la asimilación de competencias cognitivas disciplinares financieras, como lo mencionan algunos de los profesores: los estudiantes condicionan la presentación de pruebas evaluatorias y los resultados de la evaluación, a sus estados anímicos coyunturales, a sus situaciones de ayuda becaria y subsidiaria de sus empresas que les exigen un promedio de notas para ganarse la ayuda, a sus relaciones psicoafectivas que alcancen a tener con sus profesores, y a su determinación firme de calificar mal a su profesor si este le pone una calificación deficiente en el proceso de enseñanza-aprendizaje. Como lo plantea Villaroel (2012), en la evaluación sumativa se valora la nota y no el aprendizaje, creando conductas reñidas con la moral que deslegitiman el valor pedagógico y humano de la educación. 


\section{DISCUSIÓN}

\section{FALLAS DE CONTEXTO EDUCATIVO: ENFOQUE INSTITUCIONAL}

Los resultados de la investigación permiten establecer que los niveles de asimilación de competencias cognitivas disciplinares de los estudiantes de último semestre del programa administración financiera son bajo-medio, debido a la premura del tiempo para entregar tareas, lo cual, obstaculiza devoluciones creativas y prácticas reflexivas, para obtener conocimientos y habilidades que perduren en el largo plazo. Además, el estudiante se enfrenta a una abrumadora cantidad de contenidos que no dan el tiempo necesario para gestionar su administración y asimilación (Ríos C., P, 2001), creando una divergencia que se manifiesta como bajos desempeños de CCD en los discentes. En esta investigación se identificó que frente a esta situación, el estudiante no busca sus propias estrategias de aprendizaje, porque no las conocen, no se las han enseñado y no son autónomos para buscarlas, mostrando dificultades para pensar por sí mismos en sentido crítico como lo afirma Kamil (s.a), dejando al descubierto las fallas del contexto educativo.

También se agrega a las fallas de contexto educativo, la poca supervisión individual del progreso de los discentes, para asegurar que los mismos estén alcanzando el nivel adecuado en sus procesos de aprendizaje, dejando en evidencia las necesidades educativas especiales insatisfechas, y poniendo de relieve la responsabilidad de profesores, los cuales, manifiestan una pauperización de su actividad docente como lo afirma Giroux (1990), porque sus funciones tienden a ser más de gestión administrativa que formadoras y transformadoras del conocimiento y de las realidades sociales. El rol de acompañamiento individual que debe hacer el profesor al estudiante, se dificulta por la cantidad de estudiantes en un mismo curso, por el poco tiempo para desarrollar los temas, por asumir labores administrativas y nuevas responsabilidades, por desmotivaciones propias generadas por detrimento del bienestar. Según Habermas (1998), es una falla de contexto, porque no se está produciendo una acción comunicativa, la cual, incluye el objetivo del consenso o el acuerdo mediante la validez de los argumentos y no de la posición de poder que ostentan los interlocutores.

Como elemento final de las fallas de contexto educativo, los resultados alcanzados por los estudiantes en las pruebas de evaluación de Estado Saber Pro, corroboran los bajos desempeños en habilidades técnicas y conceptuales en el componente económico y financiero encontrado en la presente investigación, los cuales, contrastan con los buenos resultados de rendimiento académico que se 
muestran en los cursos objeto de estudio. Mientras en las pruebas de Estado en los Módulos de Gestión Financiera, Gestión de las Organizaciones y Formulación, Evaluación y Gestión de Proyectos, los estudiantes se ubicaron por debajo de la media, en los cursos de Análisis Financiero, Matemática Financiera y, Formulación y Evaluación de Proyectos del programa administración financiera de la universidad, los estudiantes se ubicaron por encima de la media. Siendo los contenidos de ambas evaluaciones muy semejantes, se puede interpretar que la evaluación sumativa de la universidad no es garantía para afirmar que el estudiante ha asimilado plenamente las competencias cognitivas disciplinares de los cursos en mención, toda vez que, las competencias representan una combinación dinámica de conocimientos, habilidades y capacidades financieras (Tuning, 2003), que el estudiante del programa debe ser capaz de demostrar.

\section{PATRONES DE COMPORTAMIENTO: ENFOQUE SOCIO CULTURAL}

Los resultados de la investigación ponen en evidencia que los estudiantes tienen un bajo nivel de habilidades técnicas financieras y un nivel medio de habilidades conceptuales financieras, relacionados con ciertos patrones de comportamiento como:

- La ausencia de estrategias meta cognitivas (Tesouro, 2015), que le permitan planear su aprendizaje, gestionar lo que desea aprender y autoevaluar los resultados del proceso.

- La validación de los aprendizajes a través de CCD financieras, está siendo influenciada por factores exógenos que dificultan mejorar los bajos desempeños profesionales y ocupacionales. Los estudiantes condicionan la presentación de pruebas y/o resultados de la evaluación a sus estados anímicos y de salud coyunturales, a sus situaciones de ayuda becaria y subsidiaria que reciben de las empresas donde laboran y que les exigen un promedio de notas para ganarse la ayuda, a sus relaciones psicoafectivas que alcancen a tener con sus profesores, y a su determinación firme de calificar mal a su profesor si este le pone una calificación deficiente en el proceso de enseñanza-aprendizaje.

\section{DEFICIENCIAS COGNITIVAS: ENFOQUE DISCIPLINAR}

- La investigación encontró que los estudiantes en estudio, tienen un bajo nivel de resolución de problemas financieros, debido a la poca capacidad 
desarrollada para elaborar un diagnóstico de sus propias necesidades de aprendizaje, su poco trabajo autónomo que los conlleve a desarrollar habilidades de análisis y síntesis de información. Se suma a lo anterior, la falta de conocimientos financieros previos, para asumir todo el bagaje conceptual y práctico de los temas. Es necesario, para mejorar esta situación, acudir a lo expuesto por Corbi (2003), que plantea efectuar tres fases: fase 1, que el estudiante comprenda las explicaciones, participe de discusiones y otras actividades de adquisición de información; fase2, utilizar el aprendizaje a través de ejemplos y su resolución, para que el estudiante haga analogías y pueda auto explicarse los problemas y su resolución; fase 3, la práctica continuada para incrementar la rapidez y la precisión de la ejecución.

- Los estudiantes están acostumbrados a realizar razonamientos informales sin una lógica instrumental y un lenguaje técnico apropiado (Carretero, et al, 1997). Si esta práctica continúa, las deficiencias cognitivas seguirán presentes.

\section{FACTORES AFECTIVOS Y MOTIVACIONALES: ENFOQUE PSICOLÓGICO}

Se identificó en la investigación el poco hábito de lectura del estudiante, su desmotivación y poco esfuerzo en el aprendizaje, que obligan a que los profesores realicen clases magistrales en donde siguen desempeñando el rol de distribuir contenidos, facilitando que los estudiantes sigan en una zona de confort. Todo ello produce una situación de dependencia, que va en contra de los propósitos del modelo educativo de la universidad, los cuales, procuran la autonomía educativa en el estudiante.

A pesar de la situación anterior, es muy recurrente las respuestas de los estudiantes en decir que los profesores son los responsables de todo el proceso de asimilación de competencias cognitivas disciplinares.

\section{CONCLUSIÓN}

Los resultados indican poco hábito de lectura del estudiante, su desmotivación y poco esfuerzo en el aprendizaje, que obligan a que los profesores realicen clases magistrales en donde siguen desempeñando el rol de distribuir contenidos, facilitando que los estudiantes sigan en una zona de confort. 


\section{REFERENCIAS BIBLIOGRÁFICAS}

Bautista M., R. (2013). Incertidumbre y riesgos en decisiones financieras. Bogotá - Colombia. Ecoe Ediciones.

Cantú H., I. L. (2006). La autoestima y su relación con la percepción del aprendizaje. Recuperado de: http://www.comie.org.mx/congreso/memoriaelectronica/v09/ponencias/at01/PRE1178881 879.pdf

Carretero, M., Jacott, L., Limón, M., \& López-Majón, A. (1997). Construir y enseñar las Ciencias Sociales y la Historia. Aique Grupo Editor S.A.

Corbi, R. G. (Septiembre de 2003). Adquisición de habilidades cognitivas. Factores en el desarrollo inicial de la competencia experta. (Tesis Doctoral). Alicante: Universidad de Alicante. Biblioteca Virtual Miguel De Cervantes.

Cornella, A. (2000). Cómo sobrevivir a la infoxicación. En Infonomia.com, 8. Barcelona. Recuperado de: http://www.infonomia.com/img/pdf/sobrevivir_infoxicacion.pdf

Cotton, J. W. (1989). Antecedentes históricos de la teoría del aprendizaje. Enciclopedia Internacional de la Educación. Barcelona: MBCy Vicens-Vives.

Díaz B., A. (1983). Autopercepción estudiantil sobre su papel en el aprendizaje: Análisis de un caso. Recuperado de: http://publicaciones.anuies.mx/pdfs/revista/Revista48_S2A2ES.pdf

Díaz B., A. (1993). La evaluación universitaria en el contexto del pensamiento neoliberal. Asociación Nacional de Universidades e Instituciones de Educación Superior. Recuperado de: http://www.angeldiazbarriga.com/articulos/htm_articulos/1993_laevaluacion.htm

Giroux, H. (1990). Los profesores como intelectuales, Piados: Barcelona. Págs. 171-178. Recuperado de: http://www.lie.upn.mx/docs/semopta/profesores.pdf

Guía de Orientación Saber Pro (2017). Competencias específicas: Módulo de Formulación, evaluación y gestión de proyectos. Publicación del Instituto Colombiano para Evaluación de Educación ICFES.

Guía de Orientación Saber Pro (2017). Competencias específicas: Módulo de Gestión de Organizaciones. Publicación del Instituto Colombiano para Evaluación de Educación ICFES.

Guía de Orientación Saber Pro (2017). Competencias específicas: Módulo de Gestión Financiera. Publicación del Instituto Colombiano para Evaluación de Educación ICFES.

Habermas, J. (1998): Teoría de la acción comunicativa, Vol. 1. Madrid, Taurus. (p.o. 1981).

Hernández S, R., Fernández, C. y Baptista, P. (2014). Metodología de la investigación. McGrawHill Education. Sexta Edición.

ICFES. (2016). Instituto Colombiano para el Fomento de la Educación Superior -Marco de referencia para la evaluación, ICFES. Módulo de Gestión Financiera Saber Pro. Bogotá D.C.: Ministerio de Educación Nacional. Recuperado de:_https://www.google.com/search?source $=\mathrm{hp \& ei}=\mathrm{QEhFW} 43 \mathrm{EGs} 715 \mathrm{gLrmLX} \mathrm{oDQ} \& \mathrm{q}=$ modulo + de + gestion + financiera + saber + pro $+2016 \&$ oq $=$ modulo + de + gestion + financiera $+\& g s \_l=$ psy-ab.1.3.0j0i22i30k119. 30956.36339.0.41563.30.22.0.4.4.0.271.3021.0j11j5.16.0...0...1c.1.64.psy-ab..11.19. 2855.0..0i131k1.0.bTTQFytJNyQ 
Jóver, P. (7 de mayo de 2011). El viaje del héroe - Ciencia y Espíritu. VII Congreso de Ciencia y Espíritu. [Conferencia]. Disponible en: https://www.youtube.com/watch?v=Kt1zVyopmK8

Kamil, Constance (s.a) La autonomía como finalidad de la Educación: implicaciones de la Teoría de Piaget. Secretaría de Educación y Cultura- Dirección de Currículo. Universidad de Illinois, Círculo de Chicago

López C., M. A. (2013). Aprendizaje, competencias y TIC. Aprendizaje basado en competencias. Pearson Educación de México S.A. de C. V. Primera Edición. Recuperado de: https://www.flipsnack.com/jcarloslh/aprendizaje-competencias-y-tic.html

Navarro, R., J. (2003). El rendimiento académico: concepto, investigación y desarrollo. Revista Electrónica Iberoamericana sobre Calidad, Eficacia y Cambio en Educación - REICE. Vol. I. No.2. Recuperado de: http://www.ice.deusto.es/rinace/reice/vol1n2/Edel.pdf

PEI. (2014). Proyecto Educativo Institucional Uniminuto. Coordinación Editorial Uniminuto Centro Editorial Uniminuto. Recuperado de: https://www.uniminuto.edu/documents/ 941377/1434225/Proyecto +Educativo + Institucional + 2013.pdf/849a034b-2ee8-448c9aa9-93e2cef4a317

Pérez, M.V. (Noviembre 2006). Efectos del uso de estrategias cognoscitivas en la comprensión de textos en estudiantes universitarios. (Tesis de Maestría). Universidad Católica Andrés Bello. Caracas Venezuela.

Poggioli, L. (2005). Estrategias de adquisición de conocimiento. Serie Enseñando a aprender. Caracas: Fundación Polar. Recuperado de: http://www.fpolar.org.ve/poggioli/poggioli.htm

Ramírez, J., y Martínez, E. (2016). Matemática financiera: Interés, Tasas y Equivalencias. Bogotá D.C. - Colombia. Editorial Trillas de Colombia Ltda.

Ribeiro, L. (2006). Desarrollo humano. Barcelona; Ediciones Odisea.

Ríos C., P. (2001). La aventura de aprender. Caracas, Venezuela: Cognitus, C.A., 229 págs. Recuperado de: file:///C:/Users/user/Downloads/Rese\%C3\%B1as\%20del\%20libro\%20 la\%20aventura\%20de\%20aprender.pdf

Tesouro, M. (18 de septiembre de 2015). La metacognición en la escuela: la importancia de enseñar a pensar. Aula Intelimundo Blog Educativo. Disponible en: http://blog.aula intelimundo.com/la-metacognicion-en-la-escuela-la-importancia-de-ensenar-a-pensar/

Tuning (2003). Una introducción a Tuning Educational Structures in Europe. La contribución de las universidades al proceso de Bolonia. Publicaciones de la Universidad de Deusto. Bilbao. Recuperado de: http://tuningacademy.org/wp-content/uploads/2014/02/UniversitiesContribution_SP.pdf

Uniminuto (s.f.). Guía para profesores-tutores de Uniminuto Virtual y a Distancia. Conocimientos Oportunidades para crecer. Presidente Consejo de Fundadores, Diego Jaramillo Cuartas, cjm. Recuperado de: https://www.uniminuto.edu/documents/968618/1562554/ Guia + Profesores + Tutores +2015/812c5b71-7aec-423e-ab1f-41c3e9cdfd7a

Uniminuto (2010). Proyecto curricular del programa administración financiera. Instituto de Educación Virtual y Distancia. Bogotá D.C.: Centro Editorial Uniminuto.

Villarroel, J. (2012). Las calificaciones como obstáculo para el desarrollo del pensamiento. En: Revista Sophia: Colección de filosofía en Educación. No.12. Quito: Editorial Universitaria Abya-Yala.pp. 141-152. Recuperado de: http://www.redalyc.org/html/4418/441846101009/ 
Villavicencio, L. M. (2004). El aprendizaje autónomo en la educación a distancia. LatinEduca2004.com. Primer Congreso Virtual Latinoamericano De Educación a Distancia. Recuperado de: https://s3.amazonaws.com/academia.edu.documents/34078288/55_03 Manrique_Lileya.pdf?AWSAccessKeyId=AKIAIWOWYYGZ2Y53UL3A\&Expires $=\overline{15} 32 \overline{2}$

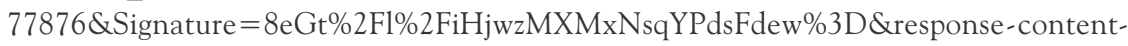
disposition=inline\%3B\%20filename\%3DAprendizaje_autonomo_de_la_educacion_a_d.pdf

Zambrano, Y. (2011). Origen etimológico de la palabra competencia. El enfoque complejo de competencias [blog]. Recuperado de: http://elenfoquecomplejodecompetencias. blogspot.com/2011/10/origen-de-la-palabra-competencias.html 